\title{
FOURIER TRANSFORMS OF SPHERICAL DISTRIBUTIONS ON COMPACT SYMMETRIC SPACES
}

\author{
GESTUR ÓLAFSSON and HENRIK SCHLICHTKRULL*
}

\begin{abstract}
In our previous articles [27] and [28] we studied Fourier series on a symmetric space $M=$ $U / K$ of the compact type. In particular, we proved a Paley-Wiener type theorem for the smooth functions on $M$, which have sufficiently small support and are $K$-invariant, respectively $K$-finite. In this article we extend these results to $K$-invariant distributions on $M$. We show that the Fourier transform of a distribution, which is supported in a sufficiently small ball around the base point, extends to a holomorphic function of exponential type. We describe the image of the Fourier transform in the space of holomorphic functions. Finally, we characterize the singular support of a distribution in terms of its Fourier transform, and we use the Paley-Wiener theorem to characterize the distributions of small support, which are in the range of a given invariant differential operator. The extension from symmetric spaces of compact type to all compact symmetric spaces is sketched in an appendix.
\end{abstract}

\section{Introduction}

The Paley-Wiener theorem for $\mathrm{R}^{n}$ describes (in the version due to L. Schwartz) the image by the Fourier transform of the space of compactly supported smooth functions on $\mathrm{R}^{n}$. A similar theorem describes the image of the space of compactly supported distributions. More precisely, let $C_{c}^{\infty}\left(\mathrm{R}^{n}\right)$ and $C_{c}^{-\infty}\left(\mathrm{R}^{n}\right)$ denote the spaces of of compactly supported smooth functions and distributions, respectively. Then the Fourier image of $C_{c}^{\infty}\left(\mathrm{R}^{n}\right)$ is the space of entire functions $F$ on $C^{n}$ of exponential type, that is, for which there exist $r>0$ and for every $N \in \mathrm{Z}^{+}=\{0,1,2, \ldots\}$ a constant $C_{N}$ such that

$$
|F(\lambda)| \leq C_{N}(1+|\lambda|)^{-N} e^{r|\operatorname{Im}(\lambda)|}
$$

for all $\lambda \in \mathrm{C}^{n}$. Furthermore, the Fourier image of $C_{c}^{-\infty}\left(\mathrm{R}^{n}\right)$ is the space of entire functions $F$ for which there exist $r>0$ and for some $N \in \mathrm{Z}^{+}$a constant $C_{N}$ such that

$$
|F(\lambda)| \leq C_{N}(1+|\lambda|)^{N} e^{r|\operatorname{Im}(\lambda)|}
$$

\footnotetext{
* The research of G. Ólafsson was supported by NSF grants DMS-0402068 and DMS-0801010. Received 27 October 2009.
} 
for all $\lambda \in \mathrm{C}^{n}$. An important aspect of these theorems is that the smallest exponent $r$ in the estimates matches with the radius of the smallest closed ball $\bar{B}_{r}=\left\{x \in \mathrm{R}^{n}|| x \mid \leq r\right\}$ containing the support of the function or distribution. Finally, by an analogous result due to Hörmander, the singular support of a compactly supported distribution is contained in $\bar{B}_{r}$ if and only if its Fourier transform $F$ satisfies the following condition. There exists $N \in \mathrm{Z}^{+}$and for every $m \in \mathrm{Z}^{+}$a constant $C_{m}$ such that

$$
|F(\lambda)| \leq C_{m}(1+|\lambda|)^{N} e^{r|\operatorname{Im}(\lambda)|}
$$

for all $\lambda \in \mathrm{C}^{n}$ with $|\operatorname{Im}(\lambda)| \leq m \log (1+|\lambda|)$. See [22], Section 1.7.

There are several generalizations of these theorems to settings where $\mathrm{R}^{n}$ is replaced by a symmetric space $X$. The most general results have been obtained for $X=G / K$ a Riemannian symmetric space of the non-compact type, by Gangolli [16] and Helgason [18], [19] for smooth functions, and by Eguchi, Hashizume and Okamoto [15] for distributions. Again the exponent matches with the radius of the support. For functions this is seen in the references just mentioned, and for distributions it is shown by Dadok [12], who gives a proof of the distributional Paley-Wiener theorem different from that of [15]. Combining these results with Hörmander's theorem for $\mathrm{R}^{n}$, a characterization of singular supports is easily deduced, see [12] (see also [1]).

In the present paper we investigate the generalization of the theorems for distributions to a Riemannian symmetric space $M=U / K$ of the compact type. In previous papers [27] and [28], we have treated the case of $K$-invariant, respectively, $K$-finite smooth functions on $M$. These papers generalized partial results in [9], [10], [17], [23]. In contrast to the non-compact cases $G / K$, the results obtained for $M$ are local in the sense that they are only valid for functions supported in sufficiently small balls, with an explicit (but not necessarily optimal) upper bound for the radius. In the present paper we use the results of [27] to extend the $K$-invariant Paley-Wiener theorem to $K$-invariant distributions on $M$, including the analogous result for singular support. The more general case of $K$-finite distributions can be treated similarly, based on [28] (the details are omitted). In an appendix at the end of the paper we briefly discuss the extension to all compact symmetric spaces of the results from [27] as well as those of the present paper.

The Paley-Wiener theorems have also been generalized to non-Riemannian symmetric spaces. General reductive symmetric spaces are treated in [7] and [8]. The case of a reductive Lie group (which can be considered as a symmetric space), was earlier treated in [5], see also [6] and [14]. Hyperbolic spaces were treated in [2]. Some partial results have been obtained for the Fourier-Laplace 
transform on causal symmetric spaces, see [3], [4], [25] and the overview in [26].

The article is organized as follows. In Section 1 we introduce the basic notation. In Section 2 we discuss the parametrization of the irreducible unitary $K$-spherical representations and the related Fourier transform. Let $f$ be a $K$ spherical smooth function (or distribution) on $M$, then its Fourier transform $\tilde{f}(\lambda)$ is defined for $\lambda$ in the semi-lattice $\Lambda^{+}(M)$ consisting of the highest weights $\lambda \in \mathfrak{a}_{c}^{*}$ of irreducible $K$-spherical representations (the weights are purely imaginary linear forms on the maximal abelian subspace $a$ ). In Section 4 we recall the main result of [27] which, in short, says the following. Assume $f$ is smooth, then the Fourier transform $\tilde{f}$ extends to a holomorphic function on $\mathfrak{a}_{\subset}^{*}$ of exponential type, and the best exponent of growth is equal to the radius of the smallest closed ball around the origin, which contains the support of $f$. Here it is required that the support of $f$ is sufficiently small, as explained in the Remark 4.3 in [27].

Section 6 contains the main results of this article. First, we introduce the Paley-Wiener space $\mathrm{PW}_{r}^{*}(\mathfrak{a})$ of holomorphic functions on $\mathfrak{a}_{\mathrm{c}}^{*}$ such that:

a) There exists $k \in \mathrm{Z}^{+}$and $C>0$ such that for all $\lambda \in \mathfrak{a}_{c}^{*}$,

$$
|\Phi(\lambda)| \leq C(1+|\lambda|)^{k} e^{r|\operatorname{Re} \lambda|}
$$

b) $\Phi(w(\lambda+\rho)-\rho)=\Phi(\lambda)$ forall $w$ in the Weyl group $W$.

Let $C_{r}^{-\infty}(M)^{K}$ denote the space of $K$-invariant distributions on $M$ with support in a closed ball of radius $r$ around the origin. Our main results are:

Theorem 6.4 (Local Paley-Wiener theorem for distributions). There exists $R>0$ such that the following holds for each $0<r<R$.

i) Let $F \in C_{r}^{-\infty}(M)^{K}$. Then the Fourier transform $\tilde{F}: \Lambda^{+}(M) \rightarrow C$ extends to a function in $\mathrm{PW}_{r}^{*}(\mathfrak{a})$.

ii) Let $\Phi \in \mathrm{PW}_{r}^{*}(\mathfrak{a})$. There exists a unique distribution $F \in C_{r}^{-\infty}(M)^{K}$ such that $\tilde{F}(\mu)=\Phi(\mu)$ for all $\mu \in \Lambda^{+}(M)$.

iii) The functions in the Paley-Wiener space $\mathrm{PW}_{r}^{*}(\mathfrak{a})$ are uniquely determined by their values on $\Lambda^{+}(M)$.

Thus, the Fourier transform followed by the extension gives a bijection

$$
C_{r}^{-\infty}(M)^{K} \simeq \mathrm{PW}_{r}^{*}(\mathfrak{a}) .
$$

THEOREM 6.6 (Characterization of singular support). Let $R$ be as above and let $0<s \leq r<R$. Let $F \in C_{r}^{-\infty}(M)^{K}$. Then the singular support of $F$ 
is contained in a closed ball of radius $s$ if and only if there exists $N \in \mathrm{Z}$ such that for each $m \in \mathrm{Z}^{+}$the holomorphic extension of $\tilde{F}$ satisfies

$$
|\tilde{F}(\lambda)| \leq C_{m}(1+|\lambda|)^{N} e^{s|\operatorname{Re} \lambda|}
$$

for all $\lambda \in \mathfrak{a}_{\mathrm{c}}^{*}$ with $|\operatorname{Re} \lambda| \leq m \log (1+|\lambda|)$.

One of the consequences of the Paley-Wiener theorem is a condition for the solvability of invariant differential equations $D T=F$ in the space $C_{r}^{-\infty}(M)^{K}$. This is stated in Theorem 6.7.

\section{Basic notation}

Let $M$ be a connected Riemannian symmetric space of the compact type. Then there exists a compact connected semisimple Lie group $U$ acting on $M$ by isometries and a closed subgroup $K \subset U$ such that $M=U / K$. Furthermore, there exists an involution $\theta: U \rightarrow U$ such that $U_{0}^{\theta} \subset K \subset U^{\theta}$. Here $U^{\theta}$ denotes the subgroup of $\theta$-fixed points, and $U_{0}^{\theta}$ its identity component. We denote the base point $e K$ in $M$ by $o$.

Let $\mathfrak{u}$ denote the Lie algebra of $U$, then $\theta$ induces an involution of $\mathfrak{u}$ (denoted by the same symbol). Let $\mathfrak{u}=\mathfrak{f} \oplus \mathfrak{q}$ be the corresponding decomposition in eigenspaces for $\theta$. Let $\langle\cdot, \cdot\rangle$ be the inner product on $\mathfrak{t}$ defined by $\langle X, Y\rangle=$ $-B(X, Y)$, where $B$ is the Killing form. We assume that the Riemannian metric $g$ of $M$ is normalized such that it agrees with $\langle\cdot, \cdot\rangle$ on the tangent space $\mathfrak{q} \simeq T_{o} M$. We denote by exp the exponential map $\mathfrak{u} \rightarrow U$, and by Exp the map $\mathfrak{q} \rightarrow M$ given by $\operatorname{Exp}(X)=\exp (X) \cdot o$. Denote by $B_{r}(0)$ the open ball in $\mathfrak{q}$ of radius $r>0$ and centered at 0 and $D_{r}(o)$ the open metric ball in $M$ of radius $r>0$ and centered at $o$. Similarly $\bar{B}_{r}(0)$ and $\bar{D}_{r}(o)$ stand for the closed balls. The exponential map Exp is surjective and an analytic diffeomorphism $B_{r}(0) \rightarrow D_{r}(o)$ for $r$ sufficiently small.

Let $\mathfrak{a} \subset \mathfrak{q}$ be a maximal abelian subspace, $\mathfrak{a}^{*}$ its dual space, and $\mathfrak{a}_{\mathfrak{C}}^{*}$ the complexified dual space. Then $\langle\cdot, \cdot\rangle$ defines an inner product on $a^{*}$. By sesquilinear extension we obtain inner products on $\mathfrak{a}_{c}^{*}$ and $i \mathfrak{a}^{*}$, which we shall denote by the same symbol. The corresponding norm is denoted by $|\cdot|$.

We denote by $\Sigma$ the set of non-zero (restricted) roots of $\mathfrak{u}_{\mathrm{C}}$ with respect to $\mathfrak{a}_{\mathrm{C}}$. Then $\Sigma \subset i \mathfrak{a}^{*}$. Furthermore, $\Sigma^{+}$stands for a fixed set of positive roots and $\rho \in i \mathfrak{a}^{*}$ denotes half of the sum of the roots in $\Sigma^{+}$counted with multiplicities. The corresponding Weyl group, generated by the reflections in the roots, is denoted $W$. 


\section{Fourier analysis on $M$}

In this section we recall the basic facts on Fourier series on $M$ and the parametrization of $K$-spherical representations. Let $\pi$ denote an irreducible unitary representation of $U$, and $V_{\pi}$ the Hilbert space on which $\pi$ acts. Let

$$
V_{\pi}^{K}=\left\{v \in V_{\pi} \mid(\forall k \in K) \pi(k) v=v\right\} .
$$

If $V_{\pi}^{K} \neq\{0\}$ then $\operatorname{dim} V_{\pi}^{K}=1$ and $\pi$ is said to be $K$-spherical. If $\pi$ is $K$-spherical, then $e_{\pi}$ will denote a fixed choice of a unit vector in $V_{\pi}^{K}$.

Recall the following parametrization of $K$-spherical irreducible representations of $U$, due to Helgason (see [20], p. 535). Denote by $\tilde{U}$ the universal covering of $U$ and by $\kappa$ the canonical projection $\tilde{U} \rightarrow U$. Then $\theta$ defines an involution $\tilde{\theta}$ on $\tilde{U}$, and the group $\tilde{K}$ of $\tilde{\theta}$-fixed points is connected. If $\pi$ is a $K$-spherical representation of $U$, then $\pi \circ \kappa$ is a $\tilde{K}$-spherical representation of $\tilde{U}$.

THeOREm 2.1. The map $\pi \mapsto \mu$, where $\mu \in i \mathrm{a}^{*}$ is the highest weight of $\pi$, induces a bijection between the set of equivalence classes of irreducible $\tilde{K}$-spherical representations of $\tilde{U}$ and the set

$$
\Lambda^{+}(\tilde{U} / \tilde{K})=\left\{\mu \in i \mathfrak{a}^{*} \mid\left(\forall \alpha \in \Sigma^{+}\right) \frac{\langle\mu, \alpha\rangle}{\langle\alpha, \alpha\rangle} \in \mathrm{Z}^{+}\right\} .
$$

For $\mu \in \Lambda^{+}(\tilde{U} / \tilde{K})$, let $\left(\pi_{\mu}, V_{\mu}\right)$ denote a fixed irreducible unitary representation of $\tilde{U}$ with highest weight $\mu$, and let $e_{\mu}=e_{\pi_{\mu}}$. We denote by $\Lambda^{+}(U / K)$ the set of elements in $\Lambda^{+}(\tilde{U} / \tilde{K})$, for which the representation $\pi_{\mu}$ of $\tilde{U}$ descends to a representation of $U$ with a $K$-fixed vector. Note that if it descends, it will have a $K_{0}$-fixed, but not necessarily a $K$-fixed vector. This was not made clear in [27], Theorem 3.1, which is only valid as stated under the extra condition that $K$ is connected. As an example take $U=\mathrm{SO}(n), n \geq 3$, and

$$
K=\mathrm{O}(n-1)=\left\{\left(\begin{array}{cc}
\operatorname{det}(A) & 0 \\
0 & A
\end{array}\right) \mid A \in O(n-1)\right\} .
$$

Then $K_{0} \simeq \mathrm{SO}(n-1)$. The natural representation of $\mathrm{SO}(n)$ acting on $\mathrm{C}^{n}$ has a $K_{0}$-fixed vector $e_{1}$, but is not $K$-spherical. However, the restricted validity does not affect the main results of [27], as the exact description of $\Lambda^{+}(U / K)$ is not used. However, the following property of $\Lambda^{+}(U / K)$ is used.

Lemma 2.2. The subset $\Lambda^{+}(U / K) \subset i \mathfrak{a}^{*}$ is closed under addition, and it has full rank in $i \mathrm{a}^{*}$, that is, there exist $\mu_{1}, \ldots, \mu_{n} \in i \mathrm{a}^{*}$ linearly independent where $n=\operatorname{dim} a$, such that

$$
\mathrm{Z}^{+} \mu_{1}+\cdots+\mathrm{Z}^{+} \mu_{n} \subset \Lambda^{+}(U / K) .
$$


Proof. It follows from Theorem 2.1 that the result holds for $\Lambda^{+}(\tilde{U} / \tilde{K})$. In fact, in this case equality is attained in (2.2) when $\mu_{1}, \ldots, \mu_{n}$ are the fundamental weights (see [34]).

Let $K^{*}=\kappa^{-1}(K)$ then $\tilde{K}=K_{0}^{*}$, and the quotient $K^{*} / \tilde{K}$ is a finite group, which acts by a homomorphism $\gamma_{\mu}: K^{*} / \tilde{K} \rightarrow$ C on the one-dimensional space $V_{\pi_{\mu}}^{\tilde{K}}$ for each $\mu \in \Lambda^{+}(\tilde{U} / \tilde{K})$. In particular, we see that $\mu$ belongs to $\Lambda^{+}(U / K)$ if and only if $\gamma_{\mu}$ is trivial. We shall see below that

$$
\gamma_{\mu+v}=\gamma_{\mu} \cdot \gamma_{v}
$$

for all $\mu, v \in \Lambda^{+}(\tilde{U} / \tilde{K})$. It follows that $\Lambda^{+}(U / K)$ is closed under addition, and also that $\gamma_{p \mu}=1$, where $p$ is the order of $K^{*} / \tilde{K}$. Hence $p \Lambda^{+}(\tilde{U} / \tilde{K}) \subset$ $\Lambda^{+}(U / K)$, and thus the lemma follows from (2.3).

For each $\mu \in \Lambda^{+}(\tilde{U} / \tilde{K})$, let $e_{\mu}=e_{\pi_{\mu}} \in V_{\mu}=V_{\pi_{\mu}}$ denote the chosen $\tilde{K}$-fixed unit vector, and let $v_{\mu} \in V_{\mu}$ be a highest weight vector normalized such that $\left\langle v_{\mu}, e_{\mu}\right\rangle=1$. Then $\int_{\tilde{K}} \pi_{\mu}(k) v_{\mu} d k=e_{\mu}$.

Consider the tensor product $V_{\mu} \otimes V_{\nu}$. It is well known that the representation $V_{\mu+v}$ occurs with multiplicity one in the tensor product, and that $v_{\mu} \otimes v_{v}$ is a highest weight vector in $V_{\mu+\nu}$. The vector

$$
e:=\int_{\tilde{K}} \pi_{\mu}(k) v_{\mu} \otimes \pi_{v}(k) v_{v} d k \in V_{\mu+v}
$$

is $\tilde{K}$-fixed. Using Fubini's theorem and the invariance of Haar measure, we see that

$$
\int_{\tilde{K}}\left(\pi_{\mu}(l) \otimes 1\right) e d l=e_{\mu} \otimes e_{\nu}
$$

In particular, $e \neq 0$ and we can identify $e$ as a multiple of the unit vector $e_{\mu+v}$. The desired relation (2.3) follows from (2.4), by using that Haar measure on $\tilde{K}$ is invariant under the adjoint action of $K^{*}$.

For $\mu \in \Lambda^{+}(M)=\Lambda^{+}(U / K)$ the spherical function associated with $\mu$ is the matrix coefficient

$$
\psi_{\mu}(x)=\left(\pi_{\mu}(x) e_{\mu}, e_{\mu}\right), \quad x \in U .
$$

It is left and right $K$-invariant and can therefore be viewed as a left $K$-invariant function on $M$. It is an eigenfunction of $\mathrm{D}(M)$, the algebra of invariant differential operators on $M$. The spherical Fourier transform of a $K$-invariant $L^{1}$-function $f$ on $M$ is the function $\tilde{f}: \Lambda^{+}(M) \rightarrow C$ defined by

$$
\tilde{f}(\mu)=\int_{M} f(x) \overline{\psi_{\mu}(x)} d x=\left(f, \psi_{\mu}\right),
$$


where $d x$ is the normalized invariant measure on $M$ (that is, $\int_{M} d x=1$ ). Notice that if $f \in L^{p}(M)$ then $|\tilde{f}(\mu)| \leq\|f\|_{p}$ as $\left|\psi_{\mu}(x)\right| \leq 1$. In particular

$$
|\tilde{f}(\mu)| \leq\|f\|_{\infty}
$$

if $f$ is continuous, and hence bounded. It follows from the Schur orthogonality relations that

$$
\tilde{\psi}_{v}(\mu)=\delta_{v, \mu} d(\mu)^{-1}
$$

for $\nu, \mu \in \Lambda^{+}(M)$, where $d(\mu)=\operatorname{dim}\left(V_{\mu}\right)$.

The spherical Fourier series for $f$ is the series given by

$$
\sum_{\mu \in \Lambda^{+}(M)} d(\mu) \tilde{f}(\mu) \psi_{\mu}
$$

Denote by $\Delta_{M}$ the negative definite Laplace operator on $M$. Then

$$
\Delta_{M} \psi_{\mu}=-\langle\mu, \mu+2 \rho\rangle \psi_{\mu} .
$$

Based on (2.7) it can be shown, see Sugiura [32], that $f$ is smooth if and only if the Fourier transform $\tilde{f}$ is rapidly decreasing, that is, for each $k \in \mathbf{Z}^{+}$there exists a constant $C_{k}$ such that

$$
|\tilde{f}(\mu)| \leq C_{k}(1+|\mu|)^{-k}
$$

for all $\mu \in \Lambda^{+}(M)$. In this case the Fourier series (2.6) converges pointwise and absolutely to $f$.

There are different ways to describe the topology on $C^{\infty}(M)^{K}$. First, the topology on $C^{\infty}(U)$ is defined by the seminorms

$$
v_{p}(f):=\left\|L_{p} f\right\|_{\infty}
$$

where $p \in U(\mathfrak{g})$. Here $L$ denotes the left regular representation and $U(\mathfrak{g})$ the universal enveloping algebra of $\mathfrak{g}$. If $\mathscr{C}$ is a closed subspace of $C^{\infty}(U)$ then the topology on $\mathscr{C}$ is given by the same family of seminorms. This applies to the space $C^{\infty}(M)$, viewed as the space of right $K$-invariant smooth functions on $U$, the space $C^{\infty}(M)^{K}$ of left $K$-invariant functions in $C^{\infty}(M)$, as well as the spaces $C_{r}^{\infty}(M)$ and $C_{r}^{\infty}(M)^{K}, r>0$, where the subscript $r$ indicates that the support is contained in $\bar{D}_{r}(o)$. Note, if $r$ is big enough then $C_{r}^{\infty}(M)=C^{\infty}(M)$.

According to [32] the topology can also be described using $\Delta_{M}$ :

Lemma 2.3. The topology of $C^{\infty}(M), C^{\infty}(M)^{K}$, and $C_{r}^{\infty}(M)^{K}$ is given by the seminorms

$$
\tau_{m}(f)=\left\|\Delta_{M}^{m} f\right\|_{\infty}, \quad m \in \mathbf{Z}^{+} .
$$


Proof. This is the corollary to Theorem 4 in [32].

We shall also need the following fact from [35] Lemma 5.6.7 or [32] Lemma 1.3.

Lemma 2.4. There exists $t_{0} \in \mathrm{R}$ such that $\sum_{\mu \in \Lambda^{+}(M)}(1+|\mu|)^{-t}<\infty$ if $t>t_{0}$.

By Weyl's dimension formula, the map $\mu \mapsto d(\mu)$ extends to a polynomial function on $\mathfrak{a}_{\mathrm{C}}^{*}$. We derive the following consequence from Lemmas 2.3 and 2.4 together with (2.7) and (2.8).

Lemma 2.5. Let $f \in C^{\infty}(M)$. Then the Fourier series (2.6) converges to $f$ in $C^{\infty}(M)$.

It follows from the $K A K$-decomposition of $U$ that the restriction map $\left.f \mapsto f\right|_{A \cdot o}$ is injective for $f \in C^{\infty}(M)^{K}$. We use the topology on $C^{\infty}(A \cdot o)$ given by the seminorms $v_{u}(f)=\left\|L_{u} f\right\|_{\infty}, u \in U(\mathfrak{a})$. Then $C^{\infty}(A \cdot o)$ is a Fréchet space, and $C^{\infty}(A \cdot o)^{W}$ is a closed subspace whose topology is given by the same family of seminorms. The following lemma gives a different way to describe the topology on $C^{\infty}(M)^{K}$ :

Lemma 2.6. The restriction map from $C^{\infty}(M)^{K}$ to $C^{\infty}(A \cdot o)^{W}$ is a topological isomorphism. It is also a topological isomorphism from $C_{r}^{\infty}(M)^{K}$ onto $C_{r}^{\infty}(A \cdot o)^{W}$, for each $r>0$.

Proof. According to [13], Theorem 1.7, the restriction map is bijective. It is obviously continuous. By the open mapping theorem for Fréchet spaces [33], Theorem 17.1., p. 170, it follows that the restriction map is a topological isomorphism. For the last statement we note first that $C_{r}^{\infty}(A \cdot o)^{W}$ is closed in $C^{\infty}(A \cdot o)^{W}$ and similarly for $C_{r}^{\infty}(M)^{K}$ in $C^{\infty}(M)^{K}$. Furthermore, the metric ball in $A \cdot o$ of radius $r$ centered at $o$ is $\bar{D}_{r}(o) \cap A \cdot o$ and $\bar{D}_{r}(o)=K\left(\bar{D}_{r}(o) \cap A \cdot o\right)$. Hence $\left.f \mapsto f\right|_{A \cdot o}$ is a bijection $C_{r}^{\infty}(M)^{K} \rightarrow C_{r}^{\infty}(A \cdot o)^{W}$, and it follows from the first statement that it is an isomorphism.

\section{The Fourier series of a distribution}

The continuous dual of $C^{\infty}(M)$, denoted by $C^{-\infty}(M)$, is the space of distributions on $M$. Recall that $U$ acts on $C^{-\infty}(M)$ by

$$
L_{g} F(f)=F\left(L_{g^{-1}} f\right), \quad g \in U, \quad f \in C^{\infty}(M), \text { and } F \in C^{-\infty}(M) .
$$

Denote by $C^{-\infty}(M)^{K}$ the space of $K$-invariant distributions on $M$. Since $C^{\infty}(M)^{K}$ is a closed subspace of $C^{\infty}(M)$, we obtain a map from $C^{-\infty}(M)$ to $\left[C^{\infty}(M)^{K}\right]^{*}$, by taking restrictions of linear forms to this subspace. Here 
$\left[C^{\infty}(M)^{K}\right]^{*}$ denotes the space of continuous linear forms on $C^{\infty}(M)^{K}$. We provide $C^{-\infty}(M)$ and $\left[C^{\infty}(M)^{K}\right]^{*}$ with the weak *-topology.

Lemma 3.1. The restriction defines a linear isomorphism

$$
C^{-\infty}(M)^{K} \simeq\left[C^{\infty}(M)^{K}\right]^{*} .
$$

Proof. The mentioned restriction map is clearly continuous. Let pr : $C^{\infty}(M) \rightarrow C^{\infty}(M)^{K}$ be the projection $\operatorname{pr}(f)(x)=\int_{K} f(k x) d k$. It is continuous, hence the transposed $\operatorname{pr}^{t}$ maps $\left[C^{\infty}(M)^{K}\right]^{*} \rightarrow C^{-\infty}(M)$ is also continuous. It is easily seen that this provides the inverse to the restriction.

Lemma 3.2. Let $F: C^{\infty}(M)^{K} \rightarrow \mathrm{C}$ be linear. Then the following statements are equivalent:

(1) $F$ is a $K$-invariant distribution.

(2) There exist $C>0$ and $m \in Z^{+}$such that

$$
|F(f)| \leq C \max _{j=1, \ldots, m}\left\|\Delta_{M}^{j} f\right\|_{\infty} \quad\left(\forall f \in C^{\infty}(M)^{K}\right) .
$$

(3) There exist $C>0$ and finitely many $u_{1}, \ldots, u_{s} \in U(\mathfrak{a})$ such that

$$
|F(f)| \leq C \max _{j=1, \ldots, s}\left\|L_{u_{j}}\left(\left.f\right|_{A \cdot o}\right)\right\|_{\infty} \quad\left(\forall f \in C^{\infty}(M)^{K}\right) .
$$

Proof. This follows from Lemmas 2.3 and 2.6.

Let $w^{*} \in W$ be such that $w^{*}\left(\Sigma^{+}\right)=-\Sigma^{+}$. Then $\mu \mapsto \mu^{*}:=-w^{*}(\mu)$ defines a bijection of $\Lambda^{+}(M)$, such that $\pi_{\mu^{*}}$ is the contragradient representation to $\pi_{\mu}$. Notice that $\overline{\psi_{\mu}}=\psi_{\mu^{*}}=\psi_{\mu}^{\vee}$ where $f^{\vee}(g)=f\left(g^{-1}\right)$. Furthermore $d\left(\mu^{*}\right)=d(\mu)$. We define the Fourier transform of a spherical distribution $F \in C^{-\infty}(M)^{K}$ by

$$
\tilde{F}(\mu):=F\left(\psi_{\mu^{*}}\right)=F\left(\psi_{\mu}^{\vee}\right) .
$$

In particular, for smooth $K$-invariant functions regarded as distributions by means of the pairing with the invariant measure, the two notions of Fourier transform agree.

Lemma 3.3. Let $F \in C^{-\infty}(M)^{K}$. Then $\mu \mapsto \tilde{F}(\mu)$ has at most polynomial growth.

PRoof. This follows from (3.1) and (2.7).

We can now write down the Fourier series for $F$. 
Lemma 3.4. Let $F \in C^{-\infty}(M)^{K}$ and $f \in C^{\infty}(M)^{K}$. Then

$$
F(f)=\sum_{\mu \in \Lambda^{+}(M)} d(\mu) \tilde{f}\left(\mu^{*}\right) \tilde{F}(\mu)
$$

with absolute convergence. In particular, the distributional Fourier transform $F \mapsto \tilde{F}$ is injective.

Proof. It follows from Lemma 2.5 that

$$
f=\sum_{\mu \in \Lambda^{+}(M)} d\left(\mu^{*}\right) \tilde{f}\left(\mu^{*}\right) \psi_{\mu^{*}}
$$

in the topology of $C^{\infty}(M)^{K}$. Since $F$ is continuous we can apply it termwise, and since $d\left(\mu^{*}\right)=d(\mu)$ we then obtain (3.4) with convergence in C. The absolute convergence follows from Lemma 2.4 , since $d(\mu)$ and $\tilde{F}(\mu)$ have at most polynomial growth with respect to $\mu$.

\section{Local Paley-Wiener Theorem for $K$-invariant functions on $M$}

We recall the main results from [27].

Definition 4.1 (Paley-Wiener space). For $r>0$ let $\mathrm{PW}_{r}(\mathfrak{a})$ denote the space of holomorphic functions $\varphi$ on $a_{c}^{*}$ satisfying the following.

a) For each $k \in \mathrm{Z}^{+}$there exists a constant $C_{k}>0$ such that

$$
|\varphi(\lambda)| \leq C_{k}(1+|\lambda|)^{-k} e^{r|\operatorname{Re} \lambda|} \quad \text { for all } \quad \lambda \in \mathfrak{a}_{c}^{*} .
$$

b) $\varphi(w(\lambda+\rho)-\rho)=\varphi(\lambda)$ for all $w \in W, \lambda \in \mathfrak{a}_{c}^{*}$.

The following is Theorem 4.2 of [27]. As pointed out in [27], Remark 4.3, the known value for the constant $R$ can be different in each part of the theorem.

THEOREM 4.2. There exists $R>0$ such that the following holds for each $0<r<R$.

i) Let $f \in C_{r}^{\infty}(M)^{K}$. Then the Fourier transform $\tilde{f}: \Lambda^{+}(M) \rightarrow C$ extends to a function in $\mathrm{PW}_{r}(\mathfrak{a})$.

ii) Let $\varphi \in \mathrm{PW}_{r}(\mathfrak{a})$. There exists a unique function $f \in C_{r}^{\infty}(M)^{K}$ such that $\tilde{f}(\mu)=\varphi(\mu)$ for all $\mu \in \Lambda^{+}(M)$.

iii) The functions in the Paley-Wiener space $\mathrm{PW}_{r}(\mathfrak{a})$ are uniquely determined by their values on $\Lambda^{+}(M)$.

Thus, the Fourier transform followed by the extension gives a bijection

$$
C_{r}^{\infty}(M)^{K} \simeq \mathrm{PW}_{r}(\mathfrak{a}) .
$$


Remark 4.3. The proof of this theorem in [27] is not entirely correct, as an error occurs in the proof of Corollary 10.2. The function $\psi(\lambda)=\varphi\left(\lambda_{1}\right) \varphi_{m}\left(\lambda_{2}\right)$, constructed in the proof of the corollary is not of exponential type $r$ as stated, but only of type $\sqrt{2} r$. This follows from the estimate $\left|\lambda_{1}\right|+\left|\lambda_{2}\right| \leq \sqrt{2}|\lambda|$, which is sharp. However, one can apply the theorem of [11] to construct an entire function $\psi$ on $\mathfrak{h}_{\mathrm{C}}^{*}$, which is of the proper exponential type, and which restricts to $\varphi$ on $a$. The rest of the proof is then unchanged.

\section{Analytic continuation of spherical functions}

We need some details from [27] concerning the analytic continuation of the spherical functions $\psi_{\mu}$ with respect to the parameter $\mu$.

Let $\bar{\Omega}$ be the closure of

$$
\Omega=\left\{X \in \mathfrak{a}|(\forall \alpha \in \Sigma)| \alpha(X) \mid<\frac{\pi}{2}\right\} .
$$

As $U$ is compact, it follows that $U$ is contained in a complex Lie group $U_{\mathrm{C}}$ with Lie algebra $\mathfrak{u}_{\mathrm{C}}$. Denote by $K_{0, \mathrm{C}}$ the analytic subgroup of $U_{\mathrm{C}}$ corresponding to $f_{c}$. Note that we are at this point not assuming that $\theta$ extends to an involution on $U_{\mathrm{c}}$. Let $\mathfrak{g}=\mathfrak{f} \oplus i \mathfrak{q} \subset \mathfrak{l}_{\mathrm{C}}$ and let $G$ be the corresponding analytic subgroup of $U_{\mathrm{c}}$, then $K_{0} \subset G$ is a maximal compact subgroup. The space $M^{d}=G / K_{0}$ is the (noncompact) dual of $U / K_{0}$. Note that the center of $G$ is contained in $K_{0}$ so $M^{d}$ is independent of the choice of the complexification $U_{\mathrm{c}}$. Let $K_{\mathrm{C}}=K K_{0, \mathrm{c}}$. Then $M, M^{d} \subset M_{\mathrm{C}}:=U_{\mathrm{C}} / K_{\mathrm{C}}$. Then $K_{\mathrm{C}}$ is a closed subgroup of $U_{\mathrm{C}}$. For each $\mu \in \Lambda^{+}(M)$ the spherical function $\psi_{\mu}$ has an analytic continuation to $M_{\mathrm{C}}=U_{\mathrm{C}} / K_{\mathrm{C}}$, denoted by the same symbol, and $\left.\psi_{\mu}\right|_{M^{d}}=\varphi_{\mu+\rho}$ where $\varphi_{\lambda}$ denotes the spherical function on $M^{d}$ with spectral parameter $\lambda$. According to [24], [29] (see also the proof due to J. Faraut in [9]) the spherical function $\varphi_{\lambda}$ on $M^{d}$ has a holomorphic extension as a $K_{\mathrm{C}}$-invariant function on $K_{\mathrm{C}} \exp (2 \Omega) \cdot o$ for every $\lambda \in \mathfrak{a}_{\mathrm{c}}^{*}$. For each $x \in K_{\mathrm{C}} \exp (2 \Omega) \cdot o$ and $\lambda \in \mathfrak{a}_{\mathrm{c}}^{*}$ we define

$$
\psi_{\lambda}(x)=\varphi_{\lambda+\rho}(x)
$$

and thus obtain an extension to $a_{\mathrm{C}}^{*}$ of the map $\mu \mapsto \psi_{\mu}(x)$ where $\mu \in \Lambda^{+}(M)$. The map $(\lambda, x) \mapsto \psi_{\lambda}(x)$ is holomorphic on the open set $a_{C}^{*} \times \exp (\Omega+i \mathfrak{a}) \cdot o \subset$ $\mathfrak{a}_{\mathrm{C}}^{*} \times A_{\mathrm{C}} \cdot o$ and it satisfies the following estimate, cf. [29], Proposition 6.1:

Lemma 5.1. There exists a constant $C$ such that

$$
\left|\psi_{\lambda}(\exp (X+i Y) \cdot o)\right| \leq C e^{\max _{w \in W} \operatorname{Re} w \lambda(X)-\min _{w \in W} \operatorname{Im} w \lambda(Y)}
$$

for all $X \in \bar{\Omega}, Y \in \mathfrak{a}$ and $\lambda \in \mathfrak{a}_{C}^{*}$. 
Corollary 5.2. Let $X_{1}, \ldots, X_{j} \in \mathfrak{a}$ and $X \in \Omega$. There exists a constant C such that

$$
\left|L_{X_{1} \ldots X_{j}} \psi_{\lambda}(\exp (X) \cdot o)\right| \leq C(1+|\lambda|)^{j} e^{|X||\operatorname{Re} \lambda|}
$$

for all $\lambda \in \mathfrak{a}_{\mathrm{c}}^{*}$. The constant $C$ depends locally uniformly on $X$.

Proof. Let $V$ be a complex neighborhood of 0 such that $X+V \subset \Omega+i a$. Let $Z_{1}, \ldots, Z_{\ell}$ be an orthonormal basis for $a$. By considering linear combinations and using that $a$ is abelian it is enough to prove the claim for the derivatives

$$
\left.\left(\frac{\partial}{\partial x_{1}}\right)^{m_{1}} \cdots\left(\frac{\partial}{\partial x_{\ell}}\right)^{m_{\ell}} \psi_{\lambda}\left(\exp \left(X+x_{1} Z_{1}+\cdots+x_{\ell} Z_{\ell}\right) \cdot o\right)\right|_{x_{1}=\cdots=x_{\ell}=0} .
$$

To simplify the notation let

$$
f_{\lambda}\left(x_{1}, \ldots, x_{\ell}\right)=\psi_{\lambda}\left(\exp \left(X+x_{1} Z_{1}+\cdots+x_{\ell} Z_{\ell}\right) \cdot o\right) .
$$

We will also use the following notation for $m=\left(m_{1}, \ldots, m_{\ell}\right) \in\left(\mathbf{Z}^{+}\right)^{\ell}$ and $\zeta=\left(\zeta_{1}, \ldots, \zeta_{\ell}\right) \in C^{\ell}: m !:=m_{1} ! \ldots m_{\ell} !,|m|=m_{1}+\cdots+m_{\ell}$, $m+1=\left(m_{1}+1, \ldots, m_{\ell}+1\right)$, and $\zeta^{m}=\zeta_{1}^{m_{1}} \ldots \zeta_{\ell}^{m_{\ell}}$. We also set $\partial^{m}=$ $\left(\partial / \partial x_{1}\right)^{m_{1}} \ldots\left(\partial / \partial x_{\ell}\right)^{m_{\ell}}$.

Let $\epsilon_{0}>0$ be so small that

$$
\left\{z_{1} Z_{1}+\cdots+z_{\ell} Z_{\ell}|| z_{j} \mid<\epsilon_{0} \text { for } j=1, \ldots, \ell\right\} \subset V .
$$

Then $f_{\lambda}$ is holomorphic on $\left\{z=\left(z_{1}, \ldots, z_{\ell}\right)|| z_{j} \mid<\epsilon_{0}\right.$ for $\left.j=1, \ldots, \ell\right\}$. By Cauchy's integral theorem for the derivatives of $f_{\lambda}$ we get for each $\epsilon<\epsilon_{0}$

$$
\partial^{m} f_{\lambda}(0)=\frac{m !}{(2 \pi i)^{\ell}} \oint_{\left|z_{1}\right|=\epsilon} \cdots \oint_{\left|z_{\ell}\right|=\epsilon} \frac{f_{\lambda}(\zeta)}{\zeta^{m+1}} d \zeta_{1} \ldots d \zeta_{\ell} .
$$

Thus (5.3) implies, with the same constant $C$ as in (5.3), that

$$
\begin{aligned}
\left|\partial^{m} f_{\lambda}(0)\right| & \leq C m !(2 \pi)^{-\ell} \epsilon^{-(|m|+\ell)} e^{|X||\operatorname{Re} \lambda|} e^{\epsilon \ell(|\operatorname{Re} \lambda|+|\operatorname{Im} \lambda|)}(2 \pi \epsilon)^{\ell} \\
& =C m ! e^{\epsilon \ell(|\operatorname{Re} \lambda|+|\operatorname{Im} \lambda|)} \epsilon^{-|m|} e^{|X||\operatorname{Re} \lambda|} .
\end{aligned}
$$

Now, take

$$
\epsilon=\frac{\epsilon_{0}}{\ell(1+|\lambda|)}
$$

then, with a new constant $C$ depending on $V$, but independent of $\lambda$ and $X$, we get

$$
\left|\partial^{m} f_{\lambda}(0)\right| \leq C(1+|\lambda|)^{|m|} e^{|X||\operatorname{Re} \lambda|}
$$

as was to be shown. 


\section{Paley-Wiener Theorem for Distributions}

In this section we state and prove the Paley-Wiener theorem for distributions on $M$.

Definition 6.1 (Paley-Wiener space for distributions). For $r>0$ let $\mathrm{PW}_{r}^{*}(\mathfrak{a})$ denote the space of holomorphic functions $\Phi$ on $\mathfrak{a}_{\mathrm{C}}^{*}$ satisfying the following.

a) There exists a $k \in \mathrm{Z}^{+}$and a constant $C_{k}>0$ such that

$$
|\Phi(\lambda)| \leq C_{k}(1+|\lambda|)^{k} e^{r|\operatorname{Re} \lambda|}
$$

for all $\lambda \in \mathfrak{a}_{c}^{*}$.

b) $\Phi(w(\lambda+\rho)-\rho)=\Phi(\lambda)$ for all $w \in W, \lambda \in \mathfrak{a}_{c}^{*}$.

Let $r>0$. A distribution $F$ has support in $\bar{D}_{r}(o)$ if and only if $F(f)=0$ for all $f \in C^{\infty}(M)$ with $\operatorname{Supp}(f) \subset M \backslash \bar{D}_{r}(o)$. Denote by $C_{r}^{-\infty}(M)$ the space of distributions that are supported on $\bar{D}_{r}(o)$.

Remark 6.2. Recall (see (3.2)) that every distribution $F$ on $M$ satisfies an estimate

$$
|F(f)| \leq C \sup _{x \in M, j \leq k}\left|\Delta_{M}^{j} f(x)\right| .
$$

If the support of $F$ is contained in some compact subset $S \subset M$, it is tempting to replace the supremum over $x \in M$ by the supremum over $x \in S$, but in general such an estimate is false. The supremum has to be taken over an open neighborhood of $S$ (see [31], example p. 95 and discussion p. 98-100). This causes a minor complication in the proof of Theorem 6.4 (this problem appears to be overlooked in [12]).

We need the following elementary result.

Lemma 6.3. Let $\Omega \subset \mathrm{C}^{\ell}$ be open and let $M$ be a differentiable manifold. Let $f \in C^{\infty}(\Omega \times M)$, and assume that $f$ is holomorphic in the first variable. Then $z \mapsto f(z, \cdot)$ is holomorphic as a map $\Omega \rightarrow C^{\infty}(M)$.

Proof. We first observe that for $a \in \Omega \subset \mathrm{R}^{2 \ell}$ and $f \in C^{\infty}(\Omega \times M)$, we have

$$
\frac{f\left(a+h e_{j}, \cdot\right)-f(a, \cdot)}{h} \rightarrow \frac{\partial f}{\partial x_{j}}(a, \cdot)
$$

in $C^{\infty}(M)$ for $h \rightarrow 0$ and $j=1, \ldots, 2 \ell$. Hence, if $T$ is a continuous linear form on $C^{\infty}(M)$, it follows that $a \mapsto T(f(a, \cdot))$ is differentiable on $\Omega$ with

$$
\frac{\partial}{\partial x_{j}}[T(f(a, \cdot))]=T\left(\frac{\partial f}{\partial x_{j}}(a, \cdot)\right) .
$$


It follows from this observation that $z \mapsto T(f(z, \cdot))$ is continuously differentiable and satisfies the Cauchy-Riemann equations, for each continuous linear form $T$ on $C^{\infty}(M)$. Hence $z \mapsto f(z, \cdot)$ is weakly holomorphic into $C^{\infty}(M)$, and, as this space is Fréchet, also strongly holomorphic.

Theorem 6.4 (Local Paley-Wiener theorem for distributions). There exists $R>0$ such that the following holds for each $0<r<R$.

i) Let $F \in C_{r}^{-\infty}(M)^{K}$. Then the Fourier transform $\tilde{F}: \Lambda^{+}(M) \rightarrow C$ extends to a function in $\mathrm{PW}_{r}^{*}(\mathfrak{a})$.

ii) Let $\Phi \in \mathrm{PW}_{r}^{*}(\mathfrak{a})$. There exists a unique distribution $F \in C_{r}^{-\infty}(M)^{K}$ such that $\tilde{F}(\mu)=\Phi(\mu)$ for all $\mu \in \Lambda^{+}(M)$.

iii) The functions in the Paley-Wiener space $\mathrm{PW}_{r}^{*}(\mathfrak{a})$ are uniquely determined by their values on $\Lambda^{+}(M)$.

Thus, the Fourier transform followed by the extension gives a bijection

$$
C_{r}^{-\infty}(M)^{K} \simeq \mathrm{PW}_{r}^{*}(\mathfrak{a}) .
$$

Remark 6.5. Note, that as in Theorem $4.2, R$ can be different in each part of the above theorem.

Proof. (i) Let $R>0$ be such that $D_{R}(o) \subset K \exp \Omega \cdot o$, where $\Omega$ is defined in (5.1). Let $r<R$ and let $\epsilon>0$ be so that $r+\epsilon<R$. Let $\varphi \in C^{\infty}(M)^{K}$ be a function which is 1 on a neighborhood of the closed ball $\bar{D}_{r}(o)$, and supported on $\bar{D}_{r+\epsilon}(o)$. The product $\varphi \psi_{\lambda}$ is a globally defined smooth function on $M$, and it belongs to $C^{\infty}(M)^{K}$ for all $\lambda \in \mathfrak{a}_{c}^{*}$. Let $F \in C_{r}^{-\infty}(M)^{K}$. We extend the Fourier transform of $F$ to a function on $a_{c}^{*}$ by

$$
\tilde{F}(\lambda):=F\left(\varphi \psi_{\lambda}^{\vee}\right) .
$$

The extension is independent of the choice of $\varphi$. Note also that $\tilde{F}(w(\lambda+\rho)-$ $\rho)=\tilde{F}(\lambda)$ and that

$$
\tilde{F}(\lambda)=F\left(\varphi \varphi_{\lambda+\rho}^{\vee}\right)=F\left(\varphi \varphi_{-\lambda-\rho}\right)=F\left(\varphi \psi_{-\lambda-2 \rho}\right) .
$$

Since the map $(\lambda, x) \mapsto \psi_{\lambda}(x)$ is smooth on the open subset $a_{\mathrm{c}}^{*} \times \exp (\Omega) \cdot o$ of $a_{c}^{*} \times A \cdot o$, it follows that $(\lambda, x) \mapsto \varphi(x) \psi_{\lambda}(x)$ is smooth on $\mathfrak{a}_{c}^{*} \times A \cdot o$. By Lemma 6.3 it follows that $\lambda \mapsto \varphi \psi_{\lambda}$ is holomorphic into $C^{\infty}(A \cdot o)$, and as it is also $W$-invariant in the $A$-variable, it follows from Lemma 2.6 that it is holomorphic into $C^{\infty}(M)^{K}$. Hence $\lambda \mapsto \tilde{F}(\lambda)$ is holomorphic on $a_{c}^{*}$.

We still need to show that this extension has exponential growth with exponent $r$. For that we will choose the function $\varphi$ of (6.1) in such a way that we 
can control the right hand side of equation (6.2) below (this is similar to what is done in the Euclidean case, see for example [22]).

As $F$ is a $K$-invariant distribution, it follows by Lemma 3.2 that there exists finitely many $u_{1}, \ldots, u_{s} \in U(\mathfrak{a})$ and a constant $C>0$, such that

$$
|\tilde{F}(\lambda)| \leq C \max _{i=1, \ldots, S}\left\|L_{u_{i}}\left(\left.\left.\varphi\right|_{A \cdot o} \psi_{\lambda}^{\vee}\right|_{A \cdot o}\right)\right\|_{\infty} .
$$

Let $m=\max _{i=1, \ldots, s} \operatorname{deg} u_{i}$. Let $h: \mathrm{R} \rightarrow \mathrm{R}$ be such that $0 \leq h \leq 1$, $\left.h\right|_{(-\infty, 1 / 3]}=1$, and $\operatorname{Supp}(h) \subseteq(-\infty, 2 / 3]$. Let $C>0$ be such that $\left\|h^{(j)}\right\|_{\infty} \leq$ $C$ for $j=0, \ldots, m$ (where $m$ is as above). Finally, for $\delta>0$ let $h_{\delta}(t)=$ $h(t / \delta)$. Then $h_{\delta}$ has the properties that

(1) $0 \leq h_{\delta} \leq 1$,

(2) $h_{\delta}(t)=1$ for all $t \leq \delta / 3$,

(3) $h_{\delta}(t)=0$ if $2 \delta / 3 \leq t$,

(4) $\left|h_{\delta}^{(j)}(t)\right| \leq C \delta^{-j}$ for all $t \in \mathrm{R}, j=0,1, \ldots, m$ and $\delta>0$.

Recall that $r+\epsilon<R$ and let $\delta \leq \epsilon$ be arbitrary for the moment. Then $r+\delta<R$. Let

$$
\varphi(x)=h_{\delta}(d(x, o)-r)
$$

for $x \in M$. Then $\operatorname{Supp}(\varphi) \subset \bar{D}_{r+\epsilon}(o)$ and $\varphi=1$ on a neighborhood of $\bar{D}_{r}(o)$. Let $j \leq m$ and let $X_{1}, \ldots, X_{j} \in \mathfrak{a}$ with $\left|X_{i}\right|=1$. By applying the chain and Leibniz rules we obtain

$$
\left|X_{1} \ldots X_{j} \varphi(x)\right| \leq C_{1} \delta^{-j}
$$

for some constant $C_{1}>0$. Note that $C_{1}$ is independent of $\delta$. In fact it only depends on the constant $C$ above, and the derivatives of $x \mapsto d(x, o)$ on the compact set $\{x \in M \mid r \leq d(x, o) \leq r+\epsilon\}$. As $d(\cdot, o)$ is smooth away from $o$ it follows that those derivatives are bounded independently of $\delta$.

For the derivatives $X_{1} \ldots X_{j} \psi_{\lambda}^{\vee}$ we note first that $|\operatorname{Re}(-\lambda-2 \rho)|=|\operatorname{Re} \lambda|$ as $\rho \in i \mathfrak{a}^{*}$. By Corollary 5.2 we get for $X \in \mathfrak{a},|X|<R$ :

$$
\left|X_{1} \ldots X_{j} \psi_{\lambda}^{\vee}(\operatorname{Exp} X)\right|=D(1+|\lambda|)^{j} e^{(r+\delta)|\operatorname{Re}(\lambda)|}
$$

for some constant $D$, independent of $\lambda$.

Using (6.1), the estimates (6.2), (6.3), (6.4), and the Leibniz rule, it follows that there exists a constant $C>0$ such that for all $\lambda \in \mathfrak{a}_{c}^{*}$ and every $\delta \leq \varepsilon$ we have

$$
|\tilde{F}(\lambda)| \leq C \delta^{-m}(1+|\lambda|)^{m} e^{(r+\delta)|\operatorname{Im} \lambda|} .
$$

We now specialize to $\delta=(1+|\lambda|)^{-1} \epsilon$ and conclude that $\tilde{F} \in \mathrm{PW}_{r}^{*}(\mathfrak{a})$. 
(ii) Let $\Phi \in \mathrm{PW}_{r}^{*}(\mathfrak{a})$. The asserted uniqueness of $F$ follows from Lemma 3.4. Motivated by (3.4) in that lemma we define $F: C^{\infty}(M)^{K} \rightarrow \mathrm{C}$ by

$$
F(f):=\sum_{\mu \in \Lambda^{+}(M)} d\left(\mu^{*}\right) \tilde{f}\left(\mu^{*}\right) \Phi(\mu) .
$$

We need to justify the convergence of the sum. Let $\omega(\lambda)=\langle\lambda, \lambda+2 \rho\rangle$. Then $\Delta_{M} \psi_{\mu}=-\omega(\mu) \psi_{\mu}$. Let $\Lambda_{1}=\Lambda^{+}(M) \backslash\{0\}$, and observe that $\omega(\mu)>0$ for all $\mu \in \Lambda_{1}$. Let $D_{1}>0$ be such that

$$
\left(\forall \mu \in \Lambda_{1}\right) \quad \omega(\mu) \geq D_{1}(1+|\mu|) .
$$

By Weyl's dimension formula, there exists a constant $D_{2}>0$ and $m \in \mathrm{Z}^{+}$ such that

$$
d\left(\mu^{*}\right) \leq D_{2}(1+|\mu|)^{m}
$$

Let $k \in \mathrm{Z}^{+}$be such that

$$
|\Phi(\lambda)| \leq C(1+|\lambda|)^{k} e^{r|\operatorname{Re} \lambda|}
$$

and let $s \in \mathrm{Z}^{+}$be such that

$$
s>m+k+t_{0}
$$

where $t_{0}$ is as in Lemma 2.4, ensuring that $\sum_{\mu \in \Lambda_{1}}(1+|\mu|)^{m+k-s}<\infty$. Using the fact that $\Delta_{M} \psi_{\mu}=-\omega(\mu) \psi_{\mu}$ we get:

$$
\begin{aligned}
\sum_{\mu \in \Lambda_{1}} d\left(\mu^{*}\right)\left|\tilde{f}\left(\mu^{*}\right)\right||\Phi(\mu)| & =\sum_{\mu \in \Lambda_{1}} d\left(\mu^{*}\right) \frac{\left|\left(\widetilde{\Delta_{M}^{s}} f\right)\left(\mu^{*}\right)\right|}{|\omega(\mu)|^{s}}|\Phi(\mu)| \\
& \leq C_{s}\left(\sum_{\mu \in \Lambda_{1}}(1+|\mu|)^{m+k-s}\right)\left\|\Delta_{M}^{s}(f)\right\|_{\infty} \\
& <\infty
\end{aligned}
$$

Here $C_{s}=C D_{1} D_{2}$. It follows that

$$
|F(f)| \leq|\Phi(0)|\|f\|_{\infty}+C_{s}\left\|\Delta_{M}^{s} f\right\|_{\infty}<\infty .
$$

Thus $F$ is well defined and continuous. It is linear by definition. It follows that $F \in C^{-\infty}(M)^{K}$. Let $\mu \in \Lambda^{+}(M)$. By application of (6.5) to $f=\psi_{\mu}^{\vee}$ it follows, using (2.5), that $\tilde{F}(\mu)=\Phi(\mu)$.

To finish the proof of (ii), we need to show $\operatorname{Supp}(F) \subseteq \bar{D}_{r}(o)$. For each $\epsilon>0$ such that $r+\epsilon<R$ let $f_{\epsilon} \in C^{\infty}(M)^{K}$ be positive with $\operatorname{Supp}\left(f_{\epsilon}\right) \subseteq$ 
$\bar{D}_{\epsilon}(o)$ and $\int_{M} f_{\epsilon}(x) d x=1$. Note that $\left|\tilde{f}_{\epsilon}(\mu)\right| \leq 1$ for all $\mu \in \Lambda^{+}(M)$ and that $\lim _{\epsilon \rightarrow 0} \tilde{f}_{\epsilon}(\mu) \rightarrow 1$ for each $\mu \in \Lambda^{+}(M)$.

Denote the holomorphic extension of $\tilde{f}_{\epsilon}$ by the same letter and recall that $\tilde{f}_{\epsilon} \in \mathrm{PW}_{\epsilon}(\mathfrak{a})$. Let $\phi_{\epsilon}(\lambda):=\Phi(\lambda) \tilde{f}_{\epsilon}(\lambda)$. Then $\phi_{\epsilon} \in \mathrm{PW}_{r+\epsilon}(\mathfrak{a})$. By Theorem 4.2, part (ii), there exists $F_{\epsilon} \in C_{r+\epsilon}^{\infty}(M)^{K}$ such that $\tilde{F}_{\epsilon}=\phi_{\epsilon}$. In particular

$$
\int f(x) F_{\epsilon}(x) d x=\sum_{\mu \in \Lambda^{+}(M)} d\left(\mu^{*}\right) \tilde{f}\left(\mu^{*}\right) \tilde{f}_{\epsilon}(\mu) \Phi(\mu)
$$

for all $f \in C^{\infty}(M)^{K}$. Hence, using (6.6) to justify the limit,

$$
\lim _{\epsilon \rightarrow 0} \int_{M} f(x) F_{\epsilon}(x) d x=F(f) .
$$

As the support of $F_{\epsilon}$ is contained in $\bar{D}_{r+\epsilon}(o)$ it follows that the support of $F$ is contained in

$$
\bigcap_{\epsilon>0} \bar{D}_{r+\epsilon}(o)=\bar{D}_{r}(o) \text {. }
$$

(iii) follows as in the proof of Theorem 4.2 in [27], given in Section 7 of that paper.

Let $F \in C^{-\infty}(M)^{K}$. The singular support of $F$, is the complement of the largest open set on which $F$ is given by a smooth function.

Theorem 6.6 (Characterization of singular support). Let $R$ be as in Theorem 4.2 and $0<s \leq r<R$. Let $F \in C_{r}^{-\infty}(M)^{K}$. Then the singular support of $F$ is contained in $\bar{D}_{s}(o)$ if and only if there exists $N \in \mathrm{Z}$ such that for each $m \in \mathrm{Z}^{+}$the holomorphic extension of $\tilde{F}$ satisfies

$$
|\tilde{F}(\lambda)| \leq C_{m}(1+|\lambda|)^{N} e^{s|\operatorname{Re} \lambda|}
$$

for all $\lambda \in \mathfrak{a}_{\mathrm{c}}^{*}$ with $|\operatorname{Re} \lambda| \leq m \log (1+|\lambda|)$.

Proof. The proof, in which Theorems 4.2 and 6.4 play the crucial roles, is similar to that of Propositions 1.3 and 1.4 in [12], which is a reduction to Hörmander's theorem for the Euclidean case.

If $D$ is a differential operator on $M$, then we define the differential operator $D^{*}$ by

$$
\int_{M} D f(x) g(x) d x=\int_{M} f(x) D^{*} g(x) d x
$$

for $f, g \in C^{\infty}(M)$. Recall also the definition of the Harish-Chandra isomorphism $\gamma^{*}: \mathrm{D}(M) \rightarrow S\left(\mathfrak{a}^{*}\right)^{W}$ from Lemma 5.1 in [27]. 
Theorem 6.7 (Solvability for distributions). Let $0<r<R$, let $F \in$ $C_{r}^{-\infty}(M)^{K}$, and let $D \in \mathrm{D}(M)$. Then there exists a $T \in C_{r}^{-\infty}(M)^{K}$ such that $D T=F$ if and only if $\lambda \mapsto \tilde{F}(\lambda) / \gamma\left(D^{*},-\mu-\rho\right)$ is entire. Moreover, in that case $T$ is unique.

Proof. The proof is the same as that of Theorem 1.8, p. 419, in [20].

\section{Appendix}

In this appendix we discuss the extension to compact symmetric spaces of the preceding results. We begin by generalizing the results from [27].

Let $U$ be a connected compact Lie group, not necessarily semisimple. As before, let $\theta$ be an involution and let $M=U / K$ where $U_{0}^{\theta} \subset K \subset U^{\theta}$. Let $Z$ denote the center of $U$. We assume that $Z \cap K=\{e\}$, since otherwise we can replace $U$ by $U / Z \cap K$, noticing that $Z \cap K$ acts trivially on $U / K$. Let $u$ denote the Lie algebra of $U$, then $\mathfrak{u}=z \oplus \mathfrak{u}^{\prime}$ where $z$ is the center of $\mathfrak{u}$ and $\mathfrak{u}^{\prime}=[\mathfrak{u}, \mathfrak{t}]$ is semisimple. As before we denote by $\theta$ also the induced involution of $\mathfrak{u}$, and by $\mathfrak{u}=\mathfrak{f} \oplus \mathfrak{q}$ the corresponding Cartan decomposition. Then $z$ and $\mathfrak{u}^{\prime}$ are $\theta$-invariant, and it follows from our assumption that $z \subset \mathfrak{q}$ and $\mathfrak{u}^{\prime} \supset$ f. Denote by $Z_{0}$ and $U^{\prime}$ the analytic subgroups of $U$ corresponding to $z$ and $\mathfrak{u}^{\prime}$. Then $U=Z_{0} U^{\prime}$ and $Z_{0} \cap U^{\prime}$ is finite. It follows that $Z_{0} \times U^{\prime}$ is a covering of $U$ by the homomorphism $(z, u) \mapsto z u$. The kernel is $D=\left\{\left(z, z^{-1}\right) \mid z \in Z_{0} \cap U^{\prime}\right\}$. Thus the covering is

$$
Z_{0} \times U^{\prime} \rightarrow U \simeq\left(Z_{0} \times U^{\prime}\right) / D
$$

The identity component $K_{0}$ of $K$ is contained in $U^{\prime}$, hence the subgroup $K^{\prime}=$ $U^{\prime} \cap K$ is a symmetric subgroup of $U$. In general $K^{\prime}$ can be a proper subgroup of $K$, in spite of the assumption that $Z \cap K=\{e\}$. Let $K^{\times} \subset Z_{0} \times U^{\prime}$ denote the preimage of $K$, then it follows that the covering above induces a covering map

$$
Z_{0} \times U^{\prime} / K^{\prime} \rightarrow U / K \simeq\left(Z_{0} \times U^{\prime}\right) / K^{\times} .
$$

We fix a maximal abelian subspace $\mathfrak{a}$ of $\mathfrak{q}$, then $z \subset \mathfrak{a}$. As before, $\Sigma \subset i \mathfrak{a}^{*}$ denotes the sets of restricted roots, and $\Sigma^{+}$denotes a subset of positive roots. We donote by $\Lambda^{+}(U / K) \subset i a^{*}$ the set of highest weights of irreducible $K$-spherical representations. It is now seen that Lemma 2.2 is valid in the generalized situation too: For $Z_{0} \times U^{\prime} / K^{\prime}$ this is a straightforward extension, otherwise we repeat the proof of the lemma, replacing $K^{*}$ with $K^{\times}$.

We define the spherical Fourier transform of a $K$-invariant function on $U / K$ as before, and for each $r>0$ we define the Paley-Wiener space $\mathrm{PW}_{r}(\mathfrak{a})$ exactly as in Definition 4.1. In particular, $W$ is the Weyl group associated with the root system $\Sigma$. We can then state: 
TheOREM 7.1. Let $U / K$ be a compact Riemannian symmetric space with assumptions as described above. Then Theorem 4.2 is valid exactly as stated in Section 4.

Proof. For $Z_{0} \times U^{\prime} / K^{\prime}$ this is a straightforward extension of the proof given in [27]. For the general case we apply the covering map above. Here it is used that every smooth function $f$ on $U / K$ supported on a sufficiently small $K$-invariant neighborhood of $e K$ lifts to a smooth function $F$ on the cover $Z_{0} \times U^{\prime} / K^{\prime}$, supported in a $K^{\prime}$-invariant neighborhood of $e K^{\prime}$ of the same size. The lifted function $F$ is $K^{\prime}$-invariant if and only if $f$ is $K$-invariant, and the Fourier transform $\tilde{F}$ of the lifted function restricts to the Fourier transform $\tilde{f}$ of the original function on $\Lambda^{+}(U / K) \subset \Lambda^{+}\left(Z_{0} \times U^{\prime} / K^{\prime}\right)$. Noticing that by definition $\mathrm{PW}_{r}(\mathfrak{a})$ is the same space in the two cases $U / K$ and $Z_{0} \times U^{\prime} / K^{\prime}$, we thus have a commutative diagram of bijective maps

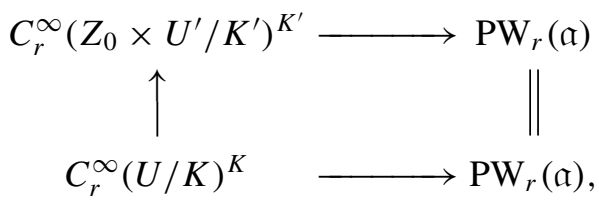

for $r$ sufficiently small. The horisontal arrows represent Fourier transform followed by holomorphic extension, and it follows from Lemma 2.2 by the argument in [27] Section 7, that functions in $\mathrm{PW}_{r}(\mathfrak{a})$ are uniquely determined by their restriction to $\Lambda^{+}(U / K)$. The theorem is now easily proved.

The main results of the present paper, Theorems 6.4 and 6.6, can now be generalized to the present setting by a straightforward extension of the previous proof. We omit the details.

\section{REFERENCES}

1. Adimurthi and Kumaresan, S., On the singular support of distributions and Fourier transforms on symmetric spaces, Ann. Scuola Norm. Sup. Pisa Cl. Sci. (4) 6 (1979), 143-150.

2. Andersen, N. B., Paley-Wiener theorems for hyperbolic spaces, J. Funct. Anal. 179 (2001), 66-119.

3. Andersen, N. B., and Ólafsson, G., A Paley-Wiener theorem for the spherical Laplace transform on causal symmetric spaces of rank 1, Proc. Amer. Math. Soc. 129 (2001), 173-179.

4. Andersen, N. B., Ólafsson, G., and Schlichtkrull, H., On the inversion of the Laplace and Abel transforms for causal symmetric spaces, Forum Math. 15 (2003), 701-725.

5. Arthur, J., A Paley-Wiener theorem for real reductive groups, Acta Math. 150 (1983), 1-89.

6. Ban, E. P. van den, and Schlichtkrull, H., Paley-Wiener spaces for real reductive Lie groups, Indag. Math. (N.S.) 16 (2005), 321-349.

7. Ban, E. P. van den, and Schlichtkrull, H., A Paley-Wiener theorem for reductive symmetric spaces, Ann. of Math. (2) 164 (2006), 879-909. 
8. Ban, E. P. van den, and Schlichtkrull, H., A Paley-Wiener theorem for distributions on reductive symmetric spaces, J. Inst. Math. Jussieu 6 (2007), 557-577.

9. Branson, T., Ólafsson, G., and Pasquale, A., The Paley-Wiener theorem and the local Huygens' principle for compact symmetric spaces: The even multiplicity case, Indag. Math. (N.S.) 16 (2005), 393-428.

10. Camporesi, R., The spherical Paley-Wiener theorem on the complex Grassmann manifolds $\mathrm{SU}(p+q) / \mathrm{S}\left(\mathrm{U}_{p} \times \mathrm{U}_{q}\right)$, Proc. Amer. Math. Soc. 134 (2006), 2649-2659.

11. Cowling, M., On the Paley-Wiener theorem, Invent. Math. 83 (1986), 403-404.

12. Dadok, J., Paley-Wiener theorem for singular support of $K$-finite distributions on symmetric spaces, J. Funct. Anal. 31 (1979), 341-354.

13. Dadok, J., On the $C^{\infty}$ Chevalley's theorem, Adv. in Math. 44 (1982), 121-131.

14. Delorme, P., Sur le théorème de Paley-Wiener d'Arthur, Ann. of Math. (2) 162 (2005), $987-$ 1029.

15. Eguchi, M., Hashizume, M., and Okamoto, K., The Paley-Wiener theorem for distributions on symmetric spaces, Hiroshima Math. J. 3 (1973), 109-120.

16. Gangolli, R., On the Plancherel formula and the Paley-Wiener theorem for spherical functions on semisimple Lie groups, Ann. of Math. (2) 93 (1971), 150-165.

17. Gonzalez, F. B., A Paley-Wiener theorem for central functions on compact Lie groups, pp. 131136 in: Radon Transforms and Tomography. Proc. South Hadley, MA 2000, Contemp. Math. 278, Amer. Math. Soc., Providence, RI 2001.

18. Helgason, S., An analogue of the Paley-Wiener theorem for the Fourier transform on certain symmetric spaces, Math. Ann. 165 (1966), 297-308.

19. Helgason, S., Paley-Wiener theorems and surjectivity of invariant differential operators on symmetric spaces and Lie groups, Bull. Amer. Math. Soc. 79 (1973), 129-132.

20. Helgason, S., Groups and Geometric Analysis, Math. Surveys and Monographs 83, Amer. Math. Soc., Providence, RI 2000.

21. Helgason, S., Differential Geometry, Lie Groups, and Symmetric Spaces, Grad. Studies in Math. 34, Amer. Math. Soc., Providence, RI 2001.

22. Hörmander, L., Linear Partial Differential Operators, Grundlehren math. Wiss. 116, Springer, Berlin 1963.

23. Koornwinder, T., A new proof of a Paley-Wiener type theorem for the Jacobi transform, Ark. Mat. 13 (1975), 145-159.

24. Krötz, B., and Stanton, R., Holomorphic extension of representations II: Geometry and harmonic analysis, Geom. Funct. Anal. 15 (2005), 190-245.

25. Ólafsson, G., and Pasquale, A., A Paley-Wiener theorem for the $\Theta$-hypergeometric transform: the even multiplicity case, J. Math. Pures Appl. (9) 83 (2004), 869-927.

26. Ólafsson, G., and Pasquale, A., Paley-Wiener theorems for the $\Theta$-spherical transform: an overview, Acta Appl. Math. 81 (2004), 275-309.

27. Ólafsson, G., and Schlichtkrull, H., A local Paley-Wiener theorem for compact symmetric spaces, Adv. Math. 218 (2008), 202-215.

28. Ólafsson, G., and Schlichtkrull, H., Fourier series on compact symmetric spaces: $K$-finite functions of small support, J. Fourier Anal. Appl. 16 (2010), 609-628.

29. Opdam, E., Harmonic analysis for certain representations of graded Hecke algebras, Acta Math. 175 (1995), 75-121.

30. Rudin, W., Functional analysis, 2nd ed., McGraw-Hill, New York 1991.

31. Schwartz, L., Théorie des distributions, Nouvelle éd., Hermann, Paris 1966.

32. Sugiura, M., Fourier series of smooth functions on compact Lie groups, Osaka Math. J. 8 (1971), 33-47.

33. Treves, F., Topological Vector Spaces, Distributions and Kernels, Pure and Appl. Math. 25, Academic Press, New York 1967. 
34. Vretare, L., Elementary spherical functions on symmetric spaces, Math. Scand. 39 (1976), 343-358.

35. Wallach, N., Harmonic Analysis on Homogeneous Spaces, Pure and Appl. Math. 19, Dekker, New York 1973.

DEPARTMENT OF MATHEMATICS

LOUISIANA STATE UNIVERSITY

BATON ROUGE

LA 70803

U.S.A.

E-mail: olafsson@math.lsu.edu
DEPARTMENT OF MATHEMATICS

UNIVERSITY OF COPENHAGEN

UNIVERSITETSPARKEN 5

DK-2100 KØBENHAVN Ø

DENMARK

E-mail: schlicht@math.ku.dk 